\title{
Utilizing a Scramble Crew Approach to Achieve 24-Hour Coverage
}

\author{
Gregory Kantor, EMT-B
}

\section{POSTER PRESENTATION ABSTRACT}

Introduction: Muhlenberg College is a small liberal arts school with a campus population of approximately 3,000. Muhlenberg College EMS (MCEMS) is a quick response service, with about 30 student volunteers and an approximate call volume of 200 emergency response calls per academic year. For 10 years, MCEMS was in service 24 hours a day; however, in 2009, MCEMS began only responding to calls from 5 p.m. until 8 a.m. during the week and 24 hours throughout the weekend, leaving the daytime unaddressed Monday through Friday. In 2016, MCEMS reinstituted daytime response, incorporating Active 911, a digital messaging system, and an all-call, scramble crew model. To our knowledge, there are no reports in the literature of collegiate-based EMS agencies incorporating this approach. Program Development \& Implementation: In daytime response during the week, MCEMS is dispatched by Muhlenberg's Department of Campus Safety through Active 911 - all members receive a notification and can indicate their availability to respond to the scene. Additionally, MCEMS crew chiefs unable to respond can indicate their availability to provide remote assistance over the phone. Program Evaluation: Since the new response plan began in March of 2016, MCEMS has responded to 47 calls that may have otherwise been ignored, at a response time shorter than that of normal duty crew responses. As part of the initiative, MCEMS has strengthened its relationship with Campus Safety through training dispatchers and adding medical supplies in all of their vehicles. Discussion/ Conclusions: Combining the scramble-crew approach with regular duty crews has both provided MCEMS' members with flexibility and, more importantly, reassured the Muhlenberg community that emergency medical services are always available on campus. Recurring training of MCEMS members and Campus Safety employees will be crucial to ensuring the model's long-term viability. MCEMS believes that this model can be beneficial to collegiate EMS agencies who wish to provide or improve daytime coverage.

Author Affiliations: Emergency Medical Services, Muhlenberg College, Allentown, PA, USA

Address for Correspondence: Gregory Kantor, EMT-B. E-mail: gk249480@muhlenberg.edu

Conflicts of Interest/Funding Sources: By the JCEMS Submission Declaration Form, all authors are required to disclose all potential conflicts of interest and funding sources. The author (G.K.) declares that they serve as a volunteer Copy Editor for JCEMS. The author declared that they have no other conflicts of interest. The authors declared that Muhlenberg College EMS received funding from Muhlenberg College's Student Activity Fee for the program described in this abstract.

Ethical Compliance: The authors attest that the research associated with this abstract was conducted in accordance with the JCEMS Ethics Guidelines.

Submission History: Received January 19, 2018; accepted for presentation and publication February 14, 2018.

Poster Presentation: This abstract was presented as a poster at the Academ- ic Poster Session of the $25^{\text {th }}$ Annual Conference of the National Collegiate Emergency Medical Services Foundation; February 24, 2018; Philadelphia, PA, USA. The poster is available for download on the JCEMS website.

Published Online: August 10, 2018

Published in Print: August 13, 2018 (Volume 1: Supplemental 1)

Reviewer Information: In accordance with JCEMS editorial policy, poster presentation abstracts undergo double-blind peer-review by at least two reviewers (JCEMS Editorial Board members and/or independent reviewers) prior to acceptance for presentation and publication. JCEMS thanks the anonymous reviewers who contributed to the review of this work.

Copyright: (C) 2018 Kantor. This is an open access abstract distributed under the terms of the Creative Commons Attribution 4.0 International (CC BY 4.0) License, which permits unrestricted use, distribution, and reproduction in any medium, provided the original author and source are credited. The full license is available at: https://creativecommons.org/licenses/ by/4.0/

Electronic Link: https://doi.org/10.30542/JCEMS.2018.01.S1.05 